\title{
Growth by $\mu-P D$ and LHPG and Characterization by Raman Spectroscopy of Potassium Lithium Niobate (KLN) Single-Crystal Fibers
}

\author{
N. Kozhaya ${ }^{*}$, M. Ferriol*, M. Cochez*, M. Aillerie*, G. Maxwelı* \\ * Laboratoire Matériaux Optiques, Photonique et Systèmes \\ ex-U.M.R. C.N.R.S. 7132, Université Paul Verlaine Metz, Supélec \\ Département Chimie I.U.T. de Moselle Est \\ 12 rue Victor Demange, BP 80105 - 57500 Saint-Avold - France \\ nader.kozhaya@univ-metz.fr \\ **Shasta Crystals, 3350 Scott Blvd., Bldg 1 (Finesse), Santa Clara, CA 95054 (USA)
}

\begin{abstract}
Homogeneous crack-free $K L N$ and Mg:KLN single-crystal fibers with the general formula $\mathrm{K}_{3} \mathrm{Li}_{2-x} \mathrm{Nb}_{5+x} \mathrm{O}_{15+2 x}$ were grown by the LHPG and $\mu$-PD techniques using pulling rates between 0.25 and $0.4 \mathrm{~mm} \cdot \mathrm{min}^{-1}$. The fibers were obtained with a diameter up to $600 \mu \mathrm{m}$ and length up to $14 \mathrm{~cm}$ for $0<x<0.3$. Their characterization by micro-Raman spectroscopy showed their homogeneous composition all along the growth axis. The evolution of the lattice vibration spectra as a function of composition was related to the increase of the structural disorder when the amount of $\mathrm{Li}_{2} \mathrm{O}$ decreases.
\end{abstract}

\section{Keywords}

KLN, Potassium Lithium Niobate, Single Crystal, Micro-Pulling Down Technique, Laser Heated Pedestal Growth, Lattice vibration, Raman spectroscopy

\section{INTRODUCTION}

Potassium lithium niobate (KLN) is a very attractive ferroelectric crystal for nonlinear optical, electro-optical and other applications [1-2]. High optical damage threshold, low optical loss, and high mechanical and chemical stability are considered to be the most important properties of KLN for nonlinear optical use. Single crystals $\mathrm{K}_{3} \mathrm{Li}_{2-x} \mathrm{Nb}_{5+x} \mathrm{O}_{15+2 x}$ $(x<0.5)$ have a tetragonal tungsten bronze type structure and are thought to be among the best candidates for second harmonic generation (SHG). Although KLN is a very attractive optical material, it is difficult to obtain high quality single-crystals because of its complicated structure and solid solution character. Tungstene bronze type structure for KLN exists only in a specific region of the ternary phase diagram $\mathrm{Li}_{2} \mathrm{O}-\mathrm{K}_{2} \mathrm{O}-\mathrm{Nb}_{2} \mathrm{O}_{5}$. The single phase subsolidus bronze (ss) region extends about from 50 to $68 \% \mathrm{Nb}_{2} \mathrm{O}_{5}$ and from 27 to $32 \mathrm{~mol} \% \mathrm{~K}_{2} \mathrm{O}$.

Local compositional variations were usually detected in the KLN crystals with higher Li content [3]. The higher the Li content is, the higher the SHG efficiency and the shorter the non-critical phase matching wavelength (NCPM) are obtained [4]. Although the mechanism has been not clarified yet, this phenomenon should be attributed to the vibration of $\mathrm{Li}^{+}$ions.

Many works were devoted to the growth of KLN single crystals, but none gave a satisfactorily interpretation of the growth results towards the only available phase diagram [5]. Thus a new exhaustive investigation of this phase diagram is required in order to relate it to the crystal growth results in the right way.

Usually, KLN single crystals can be grown by conventional methods i.e. top-seeded solution growth (TSSG) [1] and Czochralski (CZ) [6] leading to compositionnally inhomogeneous crystals due to their solid solution origin combined with low pulling rates. The purpose of this work is to optimize the quality and properties of KLN crystals by relating the growth results by two techniques: $\mu-P D$ (micropulling down) [7-9] and LHPG (laser heated pedestal growth) [10-11] and the new determination of phase equilibria relationships in the ternary system $\mathrm{Li}_{2} \mathrm{O}-\mathrm{K}_{2} \mathrm{O}-$ $\mathrm{Nb}_{2} \mathrm{O}_{5}$ which is now in progress [12].

\section{EXPERIMENTAL PROCEDURE}

The starting materials were made by using $99.99 \% \mathrm{~K}_{2} \mathrm{CO}_{3}$, $\mathrm{Li}_{2} \mathrm{CO}_{3}, \mathrm{MgO}$ and $99.95 \% \mathrm{Nb}_{2} \mathrm{O}_{5}$. MgO doping level was between 0.1 and $1 \%$. The chemicals ( $5 \mathrm{~g}$ on the whole) were thoroughly mixed by grinding them with pure ethanol in an agate mortar. The paste was dried at $100^{\circ} \mathrm{C}$ during $2-$ 3 hours and after pressed into pellets. The pellets were placed into an alumina crucible and annealed in air at $950^{\circ} \mathrm{C}$ for 30 hours. This procedure was repeated two times to ensure the complete decomposition of carbonates.

The different compositions prepared as starting melts are given in Table 1.

To optimize compositional homogeneity when pulling solid solution crystals, it is necessary to have for all species an effective distribution coefficient as close to one as possible. This can only be obtained with high pulling rates (BPS theory [13]) and so, by using growth methods with high axial thermal gradients. This is achieved in $\mu-P D$ and LHPG devices. These two techniques have proved to give fibers with a very similar quality [14]

Undoped fibers were pulled by $\mu$-PD. The powder was placed in crucibles made of a Pt plate of $0.1 \mathrm{~mm}$ in

This is an Open Access article distributed under the terms of the Creative Commons Attribution-Noncommercial License (http://creativecommons.org/licenses/by-nc/3.0/), which permits unrestricted use, distribution, and reproduction in any noncommercial medium, provided the original work is properly cited. 


\begin{tabular}{|c|c|c|c|c|}
\hline $\mathrm{x}$ in $\mathrm{K}_{3} \mathrm{Li}_{2-\mathrm{x}} \mathrm{Nb}_{5+x} \mathrm{O}_{15+2 \mathrm{x}}$ & $\mathrm{K}_{2} \mathrm{O}$ & $\mathrm{Li}_{2} \mathrm{O}$ & $\mathrm{Nb}_{2} \mathrm{O}_{5}$ & $\mathrm{MgO}$ \\
\hline 0 & 30 & 20 & 50 & 0 \\
0.19 & 30 & 18.1 & 51.9 & 0 \\
0.23 & 30 & 17.7 & 52.3 & 0 \\
0.27 & 30 & 17.3 & 52.7 & 0 \\
0.30 & 30 & 17 & 53 & 0 \\
0.19 & 30 & 18.1 & 51.9 & 0 \\
0.19 & 30 & 18.1 & 51.9 & 0.1 \\
0.19 & 30 & 18.1 & 51.9 & 0.5 \\
0.19 & 30 & 18.1 & 51.9 & 1 \\
\hline
\end{tabular}

Table 1: Compositions of starting melts ( $\mathrm{mol} \%$ )

thickness and with dimensions of $10 \times 5 \times 3 \mathrm{~mm}$. The nozzle length was in the range $1-2 \mathrm{~mm}$ and its internal diameter either 550 or $750 \mu \mathrm{m}$ for an external diameter of 750 or 900 $\mu \mathrm{m}$. The crystal diameter was controlled manually by adjusting the suitable temperatures of the crucible, afterheater and the pulling rate. $<100\rangle$ oriented strontium barium niobate (SBN) crystal was used as seed as well as $\mathrm{KLN}$ crystals with the same orientation. A pulling rate of 0.3 $\mathrm{mm} \cdot \mathrm{min}^{-1}$ was chosen and all pullings were performed under air atmosphere.

The Mg-doped fibers were grown by the LHPG method in the Shasta Crystals society. Fibers were grown under air atmosphere from ceramic rods using a $<100>$ oriented SBN seed crystal. Growth rate was in the range 0.25-0.4 $\mathrm{mm} \cdot \mathrm{min}^{-1}$.

The obtained crystals were investigated by optical microscopy (microscope LEICA DM 4000 M) and microRaman spectroscopy at room temperature using a Labram Aramis JOBIN-YVON with the following measurement conditions:

Excitation wavelength : $785 \mathrm{~nm}$

Objective: x10

Exposition time: $2 \mathrm{sec}$

Hole: 150

Grating: 1200

Mode: backscattering

\section{RESULTS AND DISCUSSION}

\subsection{Crystal growth}

Several fibers were pulled by both methods with lengths between 10 and $120 \mathrm{~mm}$ ( $\mu$-PD) (Figure 2a) and between 15 and $25 \mathrm{~mm}$ (LHPG) (Figure 2b). Under the microscope, fibers appeared clear, transparent, colorless and exempt from fractures, bubbles and inclusions. However, some defects can be noticed on the surface (Figure $2 c$ and $d$ ).

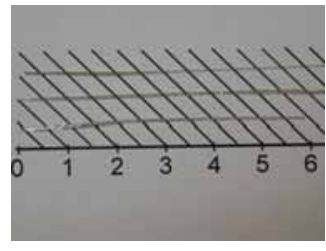

(a)

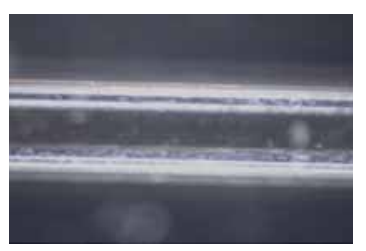

(c) $\mathrm{G}: x 50$

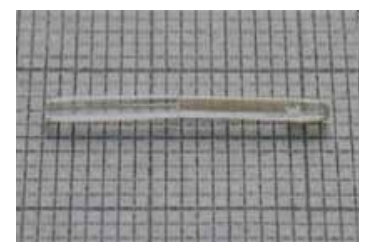

(b)

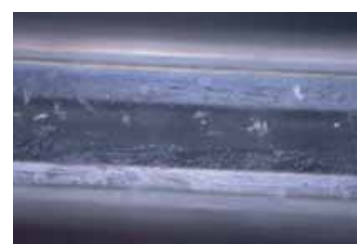

(d) G: $x 100$
Figure 2: KLN fibers obtained by $\mu-P D(a)$, LHPG (b) and under the microscope (c and d)

\subsection{Raman characterization}

\section{Raman theoretical modes}

KLN crystals have a tungsten bronze type structure with the formula $\left(A_{1}\right)_{2}\left(A_{2}\right)_{4}(C)_{4}\left(B_{1}\right)_{2}\left(B_{2}\right)_{8} O_{30}$ [15]. The structure is made of two kinds of irregular oxygen octahedra containing $\mathrm{Nb}^{5+}$ ions (B1 and B2 sites) arranged to form tunnels parallel to the c-axis delimiting three kinds of sites with triangular, square and pentagonal sections, respectively named $\mathrm{C}, \mathrm{A} 1$ and $\mathrm{A} 2$ sites. In the stoichiometric compound $(\mathrm{x}=0), \mathrm{A} 1$ and $\mathrm{A} 2$ sites are fully occupied by $\mathrm{K}^{+}$ions and $\mathrm{C}$ sites by $\mathrm{Li}^{+}$ions. $A \mathrm{XY}_{6}$ molecule (with the point symmetry $\mathrm{O}_{\mathrm{h}}$ ) has 15 internal vibrational degrees of freedom or six normal vibrational modes $v_{\mathrm{j}}$. They can be represented from group theoretical considerations as:

$A_{1 g}(R)+E_{g}(R)+2 T_{1 u}(I R)+T_{2 g}(R)+T_{2 u}$ (inactive),

The subscripts " $g$ " and " $u$ " represent symmetric and antisymmetric vibrations. Respectively, $v_{1}$ represents $A_{1 g}$, $v_{2}$ represents $E_{g}, v_{3}$ and $v_{4}$ represent $2 T_{1 u}, v_{5}$ represents $T_{2 g}$, and $v_{6}$ represents $T_{2 u} \cdot v_{1}, v_{2}$ and $v_{3}$ are all stretch vibration modes, $v_{4}, v_{5}$ and $v_{6}$ are all bend vibration modes. Thus, there should be three strong characteristic Raman peaks and two characteristic infrared reflection bands belonging to the internal vibration modes of the $\left[\mathrm{NbO}_{6}\right]^{7-}$ octahedron [16].

\section{Undoped KLN}

We observed that all Raman spectra recorded along the growth axis showed no evolution both in intensity and frequency for all the bands within measurement uncertainties (Figure 3).

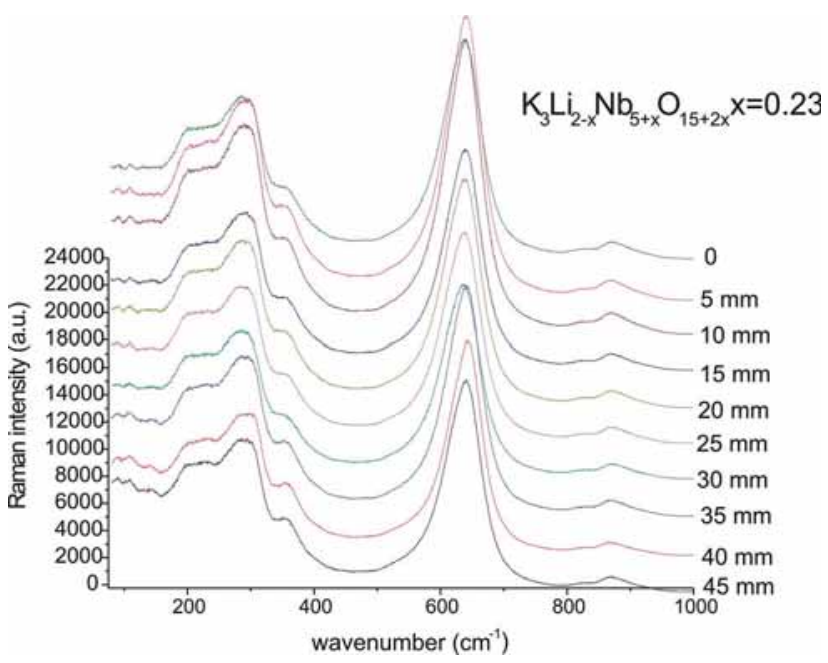

Figure 3: Typical Raman spectra for a KLN fiber $(x=0.23)$ along the growth axis

Fortin et al. showed earlier that the frequency of the Raman band near $650 \mathrm{~cm}^{-1}$, corresponding to $v_{2}$ internal modes of niobium octahedra $\left[\mathrm{NbO}_{6}\right]^{7-}$, was very sensitive to the niobium content of the crystals and then, to their stoichiometry [4] as a result of the modification of strains (octahedra more or less compressed) when the environment changes due to stoichiometry modifications. On this basis, the weak evolution (within measurement uncertainties) of the corresponding Raman frequency indicates a rather good compositional homogeneity (Figure 4).

Figure 5 shows the polarized (along the growth axis) Raman spectra of crystal fibers whose compositions are 
given in Table 1. The different peaks observed are detailed in Table 2.

The three strong Raman peaks of $\left[\mathrm{NbO}_{6}\right]^{7-}$ in the $\mathrm{KLN}$ crystal (internal vibration modes) correspond to $v_{1}, v_{2}$ and $v_{5}$. For $x=0$, they are located at 860 and $654 \mathrm{~cm}^{-1}$. The third mode $\left(v_{5}\right)$ is split into the $86 / 108 / 137 \mathrm{~cm}^{-1}$ peaks due to the deviation from the $\mathrm{O}_{h}$ symmetry and distortion of the octahedron for every $B_{1}$ and $B_{2}$ site [16]. The peaks between 162 and $539 \mathrm{~cm}^{-1}$ belong to the external vibration modes for $\left[\mathrm{NbO}_{6}\right]^{7-}$ and are presumably attributed to the $\mathrm{Li}$ ions occupying $\mathrm{C}$ sites which causes polar lattice vibrations [16].

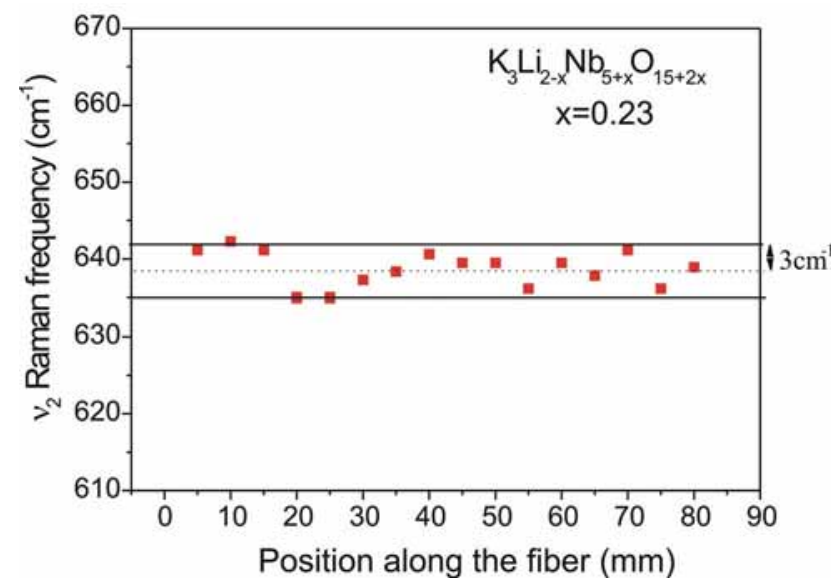

Figure 4: Evolution of $v_{2}$ Raman band near $650 \mathrm{~cm}^{-1}$ along the growth axis

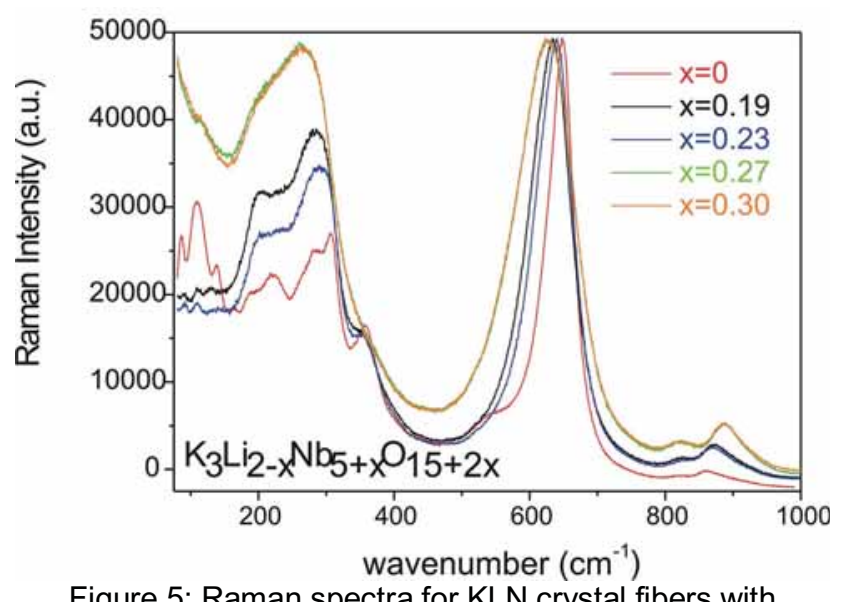

Figure 5: Raman spectra for KLN crystal fibers with different compositions polarized parallel to the growth axis

\begin{tabular}{|l|c|c|c|c|}
\hline $\mathrm{X}$ & $\left.\begin{array}{c}\mathrm{v}_{1} \\
\left(\mathrm{~cm}^{-}\right. \\
1\end{array}\right)$ & $\left.\begin{array}{c}\mathrm{v}_{2} \\
\left(\mathrm{~cm}^{-}\right. \\
1\end{array}\right)$ & $\begin{array}{c}\mathrm{v}_{5} \\
\left(\mathrm{~cm}^{-1}\right)\end{array}$ & $\begin{array}{c}\text { External vibration } \\
\text { modes }\left(\mathrm{cm}^{-1}\right)\end{array}$ \\
\hline 0 & 860 & 654 & $86 / 108 / 137$ & $\begin{array}{c}162 / 188 / 218 / 264 / 281 / \\
300 / 307 / 357 / 539\end{array}$ \\
\hline 0.19 & 875 & 634 & 108 & $200 / 253 / 284 / 353$ \\
\hline 0.23 & 870 & 640 & 107 & $201 / 256 / 286 / 354$ \\
\hline 0.27 & 885 & 624 & 110 & $\sim 200 / 260 / 290 / 355$ \\
\hline 0.3 & 885 & 624 & 110 & $\sim 200 / 260 / 290 / 355$ \\
\hline
\end{tabular}

Table 2: Raman peaks for KLN crystal fibers with different compositions.

From $x=0.19, v_{5}$ mode corresponds to a single broad peak at $108-110 \mathrm{~cm}^{-1}$. From $\mathrm{x}=0.23$, the number of external modes decreases to 4 between 162 and $540 \mathrm{~cm}^{-1}$. For $x=0.27$ and $x=0.3$, the width of all the Raman peaks significantly increases, especially in the low frequency domain (between 100 and $325 \mathrm{~cm}^{-1}$ ). For $x>0.23$, all peaks tend to disappear between 325 and $600 \mathrm{~cm}^{-1}$, the $v_{2}$ mode moves towards the red domain of frequencies and the $v_{1}$ mode moves abruptly towards the blue range. The splitting of $v_{5}$ mode and the complicated external modes in the wavelength range from 160 to $540 \mathrm{~cm}^{-1}$ shows the striking influence of $\mathrm{Li}$ ions in $\mathrm{C}$ sites on the vibrations of $\left[\mathrm{NbO}_{6}\right]^{7}$ when $x$ increases [16].

Figures 6 and 7 give the evolution of respectively the full width at half maximum (FWHM) of the $v_{2}$ mode and its frequency with the composition expressed as $\mathrm{x}$. According to Abrahams et al. [15], the observed evolution can be related to the increase of the structural disorder with $x$ which can be traduced by the formula:

$\left(\mathrm{K}_{1-\mathrm{u}} \mathrm{Li}_{\mathrm{u}}\right)\left(\mathrm{K}_{2-\mathrm{y}} \mathrm{Li} \mathrm{y}\right)\left(\mathrm{Li}_{2-5 z} \mathrm{Nb}_{\mathrm{z}} \square_{4 z}\right) \mathrm{Nb}_{5} \mathrm{O}_{15}$

It corresponds to the formation of defects: Li-antisites in $\mathrm{K}^{+}$ sites ( $\mathrm{A} 1$ and $\mathrm{A} 2$ ), Nb-antisites in $\mathrm{Li}^{+}$sites (C) and charge compensation vacancies. Figure 8 shows that the vacancies, $\mathrm{Li}$ and $\mathrm{Nb}$ antisites calculated concentrations increase with $x$ and therfore the disorder which undergoes the broadening of all the Raman peaks, particularly that of the $v_{2}$ mode and the evolution experimentally observed for the Raman spectra.

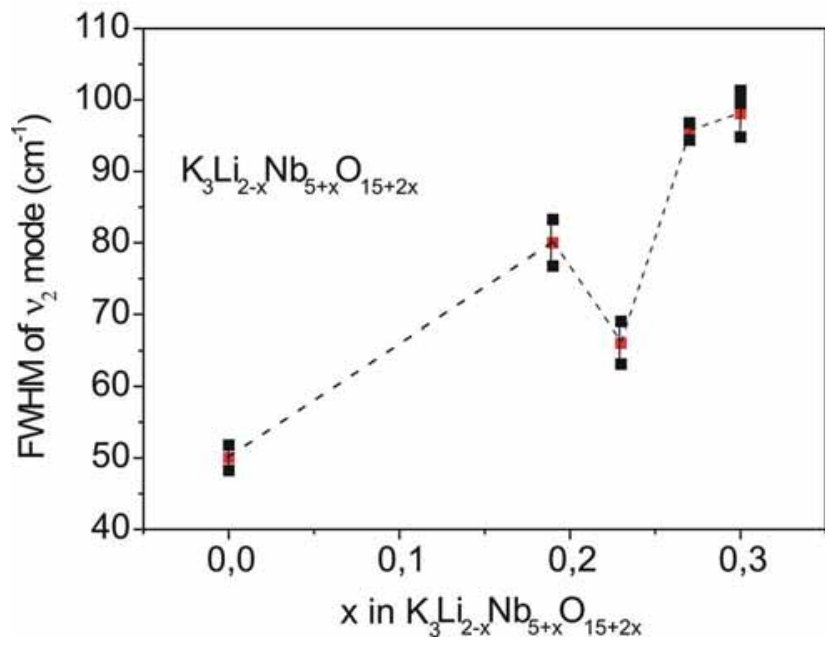

Figure 6: Evolution of FWHM of $v_{2}$ mode with composition for KLN crystal fibers

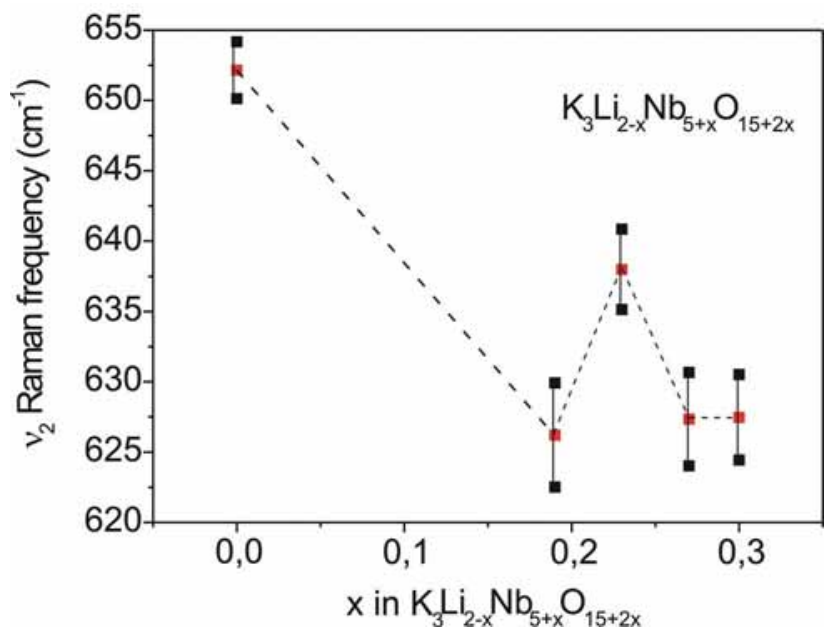

Figure 7: Evolution of Raman frequency of $v_{2}$ mode with composition for KLN crystal fibers 


\section{KLN doped with $\mathrm{Mg}^{2+}$}

Figure 9 shows Raman spectra obtained for KLN fibers doped with various concentrations of $\mathrm{Mg}^{2+}$ and grown by the LHPG method. Until $0.5 \% \mathrm{MgO}$, the shape of the Raman spectra does not particularly changes, but beyond this concentration an enlargement of the peaks of the Raman spectra can be seen. This traduces the appearance of a significant disorder caused by the introduction of magnesium ions which would preferably substitute $\mathrm{K}^{+}$ and/or $\mathrm{Li}^{+}$ions taking in account their relative size, this substitution being accompanied by the formation of compensation charge vacancies.

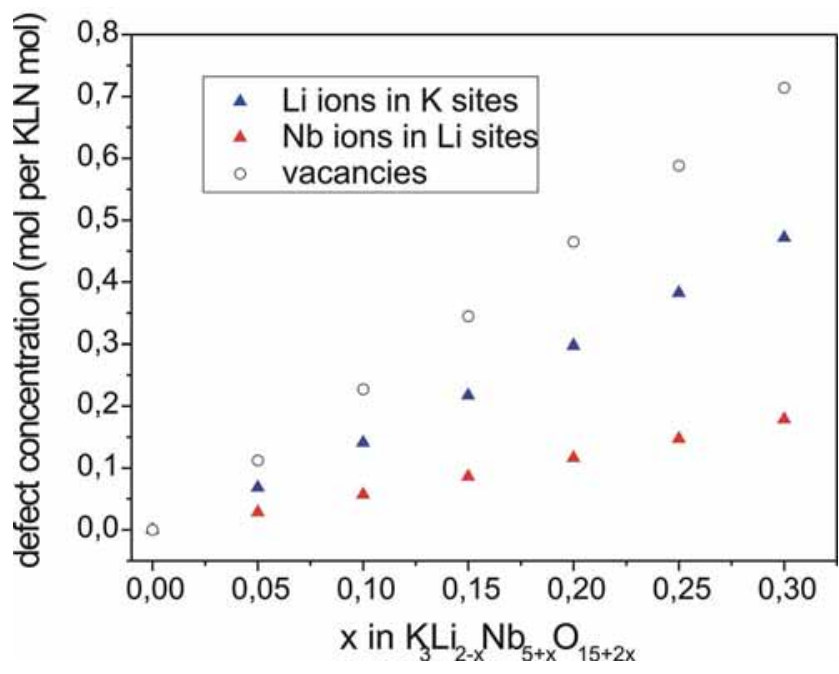

Figure 8: Evolution of the defect concentrations with composition according to Abraham's model

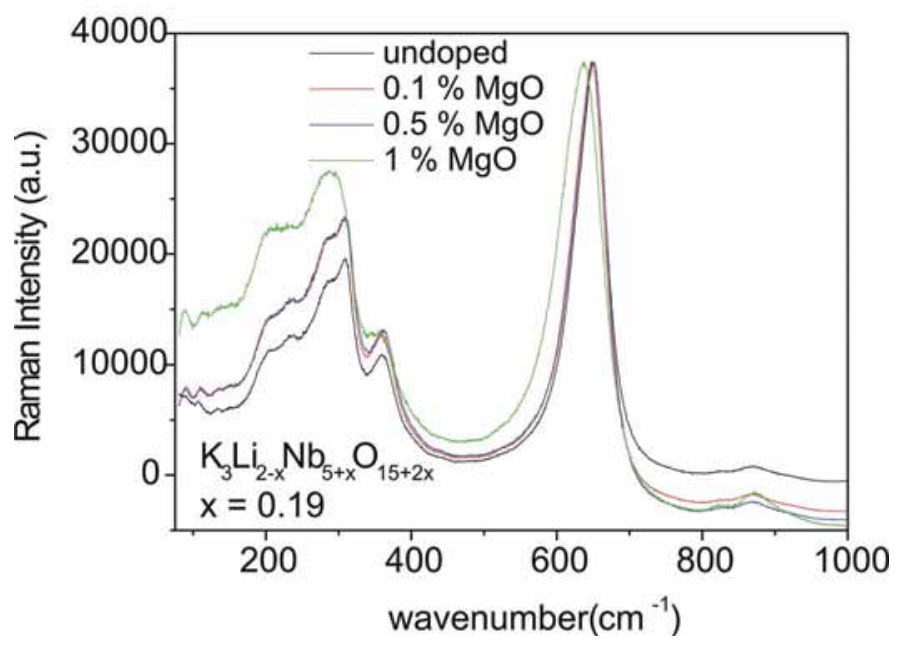

Figure 9: Raman spectra of magnesium-doped KLN singlecrystal fibers with $\mathrm{x}=0.19$

\section{CONCLUSION}

Undoped and magnesium-doped good quality KLN singlecrytal fibers with various compositions were successfully grown by $\mu-P D$ and LHPG techniques. All the fibers were characterized by optical microscopy and micro-Raman spectroscopy. It appears that the Raman spectra corresponding to the internal and external vibration modes of $\left[\mathrm{NbO}_{6}\right]^{7-}$ octahedra are strongly influenced by the changing composition of the crystals. For the $v_{2}$ mode, the frequency shift towards the red and the increase of FWHM could be related to the increase of the structural disorder introduced by the increase of $x$ (Li concentration decrease) involving charge compensation vacancies, $\mathrm{Li}$ and $\mathrm{Nb}$ antisites according to the model of Abrahams et al. [15].

\section{REFERENCES}

[1] Uitert L.G., Singh S., Levinstein H.J., Geusic J.E., Bonner W.A., 1967, Appl. Phys. Lett. 11, 161

[2] Fukuda T., 1969, Jpn. J. Appl. Phys. 8, 122

[3] Pan S.K., Yuan Q.X., Xu J., Yu T.Y., Yu B.K., Wan Y.B., 2001, J. Cryst. Growth 223, 389

[4] Fortin W., Kugel G.E., Rytz D., 1997, Ferroelectrics 202, 183

[5] Scott B.A., Giess E.A., Olson B.L., Burns G., Smith A.W., O'Kane D.F., 1970, Mat. Res. Bull. 5, 47

[6] Bonner W.J., Grodkiewicz W.H., Van Uitert L.G., 1967, J. Cryst. Growth 1, 318

[7] Epelbaum B., Schierning G., Winnacker A., 2005, J. Cryst. Growth 275, e867

[8] Chani V.I., Nagata K., Fukuda T., 1998, Ferroelectrics, 218, 9

[9] Chani V.I. in Shaped Crystals, Growth by Micro-Pulling Down Technique, Advances in Materials Research, T. Fukuda and V.I. Chani Eds., Springer-Verlag, Berlin, 2007, p. 69

[10] Matsukura M., Chen Z., Adachi M., Kawabata A., 1997, J. Appl. Phys. 36, 5947

[11] Matsukura M., Karaki T., Takeyema T., Fujii M., Adachi M., 1999, J. Appl. Phys. 38, 5638

[12] Ferriol M., Cochez M., Aillerie M., 2009, J. Cryst. Growth 311, 4343

[13] Burton J.A., Prim R.C., Slichter W.P., 1953, J. Chem. Phys., 21, 1987

[14] Rudoplh P., Fukuda T., 1999, Cryst. Res. Technol. 34,3

[15] Abrahams S.C., Jamieson P.B., Bernstein J.L., 1971, J. Chem. Phys. 54, 2355

[16] Wan Y., Guo X., Chen J., Yuan X., Chu J., Li J., 2002,

J. Cryst. Growth 235, 248 